\title{
Responsabilidad social en las instituciones de enseñanza universitaria de criminología
}

\section{Social responsibility in university teaching institutions of criminology}

Wael Sarwat Hikal Carreón ${ }^{1}$

\section{Resumen}

La investigación y la educación son partes fundamentales en todas las sociedades para el mejoramiento de las condiciones de bienestar, reconstrucción del caos social y de las circunstancias que así lo demanden; así como para el desarrollo profesional de sus egresados desde enfoques económicos, de identidad, satisfacción, entre otros. Para dar lugar a estos objetivos, los centros escolares en criminología tienen la responsabilidad de formar personas con alto sentido humanista, compromiso social, ético, con conocimientos necesarios para atender las demandas sociales y laborales, lo que les permitirá en el ejercicio de su función atender dos situaciones mencionadas: El impacto social y el desarrollo personal como profesional.

Palabras clave: Criminología; Investigación; Pertinencia de la educación; Responsabilidad (educación).

1 Doctorando en Filosofía con acentuación en Estudios de la Educación por la Facultad de Filosofía y Letras (becario CONACYT) de la Universidad Autónoma de Nuevo León. E-mail: wael.hikalcrr@uanl.edu.mx 


\section{Abstract}

Research and education are fundamental parts in all societies for the improvement of conditions, well-being, reconstruction of social chaos, and the conditions that society demands, on the other hand, as well as for the professional development of their graduated, on issues of economic, identity, satisfaction, among others. To give rise to these objectives, schools in criminology have the responsibility to train people with high humanist sense, social commitment, ethics, with necessary knowledge to meet social and employee demands, so that will allow them in the exercise of their function, to attend two situations: the social impact and personal development as a professional.

Keywords: Criminology; Research; Relevance of education; Responsibility (education).

\section{Introducción}

Se apertura esta introducción con algunas cuestiones: ¿Cuál es la responsabilidad social de los centros de educación en criminología? ¿De qué son responsables? ¿Ante quiénes responden? ¿Cuáles son los criterios de responsabilidad? (Quintana García, Benavides Velasco, y Marchante Lara, 2015). La razón de ser de los centros de educación superior reside en la transmisión de conocimientos a los educandos (Barffusón, 2015), para que éstos, en un futuro inmediato, aporten propuestas transformadoras. Las universidades han ido fragmentando en facultades los estudios que corresponden a las áreas del conocimiento para la formación de profesionales, también ocurre los mismo respecto de institutos o centros particulares de enseñanzas específicas, concretadas y centradas a ciertos estudios. En ambos casos, son las facultades o centros de educación superior que imparten criminología a los que les corresponde la transferencia y formación en conocimientos integrales (Massé, 2007) que permitan entender el problema de la criminalidad, y la solución mediante políticas públicas de seguridad y vinculación laboral para el desarrollo personal y económico (Cantú Mendoza, 2015). 
Todo esto ocurre en un proceso de responsabilidad social (Quintana García, Benavides Velasco, y Marchante Lara, 2015) de los centros de educación superior o de enseñanza de la criminología, mediante el establecimiento de sus objetivos internos (intramuros), como institución, y el engranaje de los elementos que le permitirán funcionar. Por otra parte, esas herramientas están en función de preparar profesionales aptos en conocimientos, y con compromiso por dar resultados a la sociedad (extramuros) (Barffusón, 2015), por medio del entendimiento profundo de los complejos problemas sociales, que permita la construcción de una propuesta para ofrecer soluciones al respecto; sistematizar sus estudios, investigaciones y prácticas, para transmitirlas y replicarlas, aprendiendo de las experiencias de otros, lo cual corresponde la gestión universitaria (Iñigo Bajos y Sosa Castillo, 2015).

Otra parte importante, como complemento basificador de la formación, generación del conocimiento y pertinencia social (Iñigo Bajos y Sosa Castillo, 2015) es la investigación para su especialización, forjada sobre los fenómenos que suceden en la sociedad, para identificarlos, desentrañarlos, comprender sus características y particularidades, en la medida que desde su comprensión, se podrán articular propuestas para el cambio e intervención, y además aportar la capacidad de enriquecer el corpus intelectual del criminólogo para actuar ante las demandas de la sociedad, lo que le lleva a una mejor preparación en el valor correspondiente a su desarrollo profesional, personal y económico. Todos estos factores son referentes básicos para la apropiación de una criminología.

\section{La responsabilidad social en los centros de educación en criminología}

La criminología tiene en sus planes el transformar a la sociedad y a los sujetos, disminuir la congestión criminógena o de riesgo que afecta a los individuos, y trabajar en propuestas que permitan incidir donde así se amerite (Barffusón, 2015), en políticas económicas, sociales, educativas, familiares, laborales, de asistencialismo, de desarrollo, entre tantas que, conjugadas de manera desequilibrada, im- 
pactan de forma negativa. Lograr lo anterior parte de una base única que es la educación (Massé, 2007), pues cualquier incidencia que se pretenda tener en la sociedad y en los individuos comienza por la educación en el área de conocimiento y respecto de las técnicas desde las cuales queremos abordar el mismo, para entender, explicar e intervenir un tema, lo cual yace a posteriori, en la responsabilidad social de los actores profesionales (Cantú Mendoza, 2015).

Existe algo llamado "vinculación”, que alude a la relación que existe entre las universidades y la sociedad, considerada como un elemento positivo y una virtud relacionada con el entorno (Quintana García, Benavides Velasco, y Marchante Lara, 2015). Esta vinculación contribuye a la formación de los alumnos, actualización e investigación para la solución de los problemas locales, en la mejora de los procesos de intervención social (Vences Esparza, Juárez Villalobos y Flores Alanis, 2015). La sociedad en la que estamos inmersos está abrumada de problemas criminales, y requiere constante actualización para intervenir de manera preventiva e inmediata cuando ocurre un problema, de ahí la importancia de la vinculación de los centros educativos con la sociedad (Barffusón, 2015).

La responsabilidad social de los centros educativos emana del compromiso de la formación técnica, científica y ética (para orientar el actuar profesional), así como de la sensibilidad humana y preparación de los estudiantes, de modo integral "que permita a la institución intervenir en el entorno con la suficiencia necesaria para generar dinámicas de cambio hacia una sociedad más justa y solidaria” (Vences Esparza, Juárez Villalobos y Flores Alanis, 2015, p. 69), que además de la formación, facilite los conocimientos para transformar por medio de propuestas útiles. La clave para la correspondencia de responsabilidad encuentra cabida en los siguientes ámbitos:

Gestión, investigación y extensión (Vences Esparza, Juárez Villalobos y Flores Alanis, 2015; Gaete Quezada, 2015; Iñigo Bajos y Sosa Castillo, 2015). Elementos o esferas mismas que pueden ser adopta- 
das y puestas en marcha por los centros que forman criminólogos. En este apartado, se desarrolla el referente a la gestión y extensión, mientras que se considera en otro segmento el de investigación (ligada a la extensión y a la vinculación con el entorno; es decir, a una investigación socialmente pertinente).

1. La gestión como clave para la correspondencia de responsabilidad social en la criminología

En ese sentido, la gestión tiene como objetivo relacionar la estructura, la gente, las capacidades y las políticas (Cantú Mendoza, 2015), con los fines para los cuales está constituido el centro educativo, entre los que se encuentra la generación de productos desde sus procesos y la formación de criminólogos capacitados en las más contemporáneas teorías y técnicas para la atención de los conflictos sociales, respecto de lo cual, la obligatoriedad de la escuela está en proporcionar todos los elementos en función de lograrlo (Barffusón, 2015). En las próximas líneas, se desarrollarán los componentes de tal gestión, refiriéndose a la institución, el personal y las estrategias, que se complementan y definen de manera coordinada.

Así pues, la escuela deberá dimensionar los sucesos para los cuales está formando criminólogos, pues a pesar de los graves hechos criminales que ocurren todos los días, las escuelas públicas no han tomado la corresponsabilidad social de incorporar la criminología en los planes de estudio de su oferta académica, siendo escasas las que sí lo tienen (Giménez-Salinas, 2009). Cabe la siguiente reflexión: "La educación superior debe reforzar sus funciones de servicio a [...] actividades encaminadas a erradicar la pobreza, la intolerancia, la violencia, el analfabetismo, el hambre, el deterioro del medioambiente [...] mediante un planteamiento interdisciplinario y transdisciplinario” (Barffusón, 2015, p. 121), objetivos mismos que busca incansablemente la criminología. Un centro escolar comprometido produce conocimiento científico responsable y pertinente (Barffusón, 2015). 
Las escuelas privadas que tomen en su quehacer la formación de criminólogos adquieren la responsabilidad que la escuela pública no ha adquirido, por lo tanto, en su plan organizacional, tienen que constituir instalaciones, y contratar personal capacitado para direccionar los fines hacía el beneficio social (impacto) y la vinculación laboral a sus egresados (desarrollo profesional) (Quintana García, Benavides Velasco, y Marchante Lara, 2015); e igualmente crear estructuras que faciliten el aprendizaje, como laboratorios para experimentar, bibliotecas con abundancia de materiales (puesto que el Internet no es una universidad), recursos didácticos variados, computadoras con sistemas de exploración de información, bases de datos útiles, actualizadas, aulas, y auditorios bien dotados de herramientas de comunicación.

El personal es una parte fundamental para la consecución de los objetivos de los centros escolares, que está comprometido con la realización de la labor no sólo por asuntos o cuestiones asociadas internamente a la institución, sino a la consigna del impacto que su trabajo también tendrá en la sociedad (Iñigo Bajos y Sosa Castillo, 2015). Su responsabilidad, además de administrativa para lograr que todos los ensambles avancen en función, podrá facilitar los procesos para la fluida consecución de los objetivos, conociendo bien qué es lo que puede hacer, y hacerlo del mejor modo. Por lo tanto, adquiere dos responsabilidades ya dichas, una con la institución y otra con la sociedad.

Aquello se establece con un marco de referencia, una guía (política de sistemas administrativos) que sentarán las bases de operatividad de cada área, puesto o función, que encamine a los resultados deseados con conocimiento claro de lo que se puede y no se puede hacer, y de lo que se puede mejorar o pretender como fin constante (Massé, 2007); esto es, cumplir con la misión previamente definida, lo que implica una sana gobernanza organizacional (Vences Esparza, Juárez Villalobos y Flores Alanis, 2015). El ideal es que exista una articulación entre todos los postulados que se plantean en la escuela, desde las normas internas, hasta los planes de estudio que correspondan a la realidad del contexto y las necesidades sociales (Gaete Quezada, 2015). 
Dentro de su política de vinculación, también está el intercambio de comunicación entre escuelas, ya sea de criminología, o de otras áreas donde se conecten los intereses de estudio en lo criminal, para provocar la colaboración entre éstas con una sinergia de saberes que permita el aprendizaje sobre mejores prácticas, investigaciones, resultados sobre intervenciones que hayan tenido los alumnos o docentes en las múltiples actividades durante su ejercicio profesional o en el servicio social asistencialista (Iñigo Bajos y Sosa Castillo, 2015). Por otro lado, establecer canales de divulgación del conocimiento (Barffusón, 2015), y que éste llegue a diversos sectores de la sociedad, siendo el empresarial uno muy importante para la posterior contratación de los egresados de criminología, además del sector público, que supone ser el principal interesado en reducir las repercusiones de la criminalidad en la sociedad. Es decir, trabajar canales y espacios que posibiliten la inserción de los egresados en el medio laboral.

Un efecto reverso negativo de no atender a la gestión universitaria produciría una insuficiencia en la calidad académica y formativa, un crecimiento inusitado, descoordinado y desequilibrado de las escuelas privadas, no sustentado en atender las necesidades sociales (Iñigo Bajos y Sosa Castillo, 2015), sino para beneficios comerciales, débil vinculación con los sectores sociales, insuficiencia de recursos económicos en los centros educativos, disminución de la credibilidad de los profesionales y de las instituciones, y merma de las capacidades (Barffusón, 2015, pp. 130 y 131).

En todo caso "es necesario realizar los esfuerzos necesarios para fortalecer el comportamiento socialmente responsable de las universidades" (Gaete Quezada, 2015, p. 154). De tal modo, las tareas por desarrollar son:

\footnotetext{
Mejorar la calidad académica promoviendo la responsabilidad, los valores y el compromiso social; diseñar planes de estudio orientados a la formación de valores; fortalecer la identidad de las universidades como instituciones con firmes principios de responsabili-
} 
dad social; consolidar foros y seminarios encaminados a analizar, desde la perspectiva ética, la incidencia de las propuestas universitarias en la solución de los problemas que enfrenta la región, y traducir la responsabilidad social en la búsqueda del bienestar integral, del desarrollo humano y de la sustentabilidad (Iñigo Bajos y Sosa Castillo, 2015, p. 171).

Son tres los compromisos (Quintana García, Benavides Velasco, y Marchante Lara, 2015) que pueden adoptar los centros de enseñanza en criminología:

1.1. Compromiso con el alumnado: De la escuela depende su formación, y también de sí mismo, como se expuso anteriormente, con personal apto para lograr los objetivos de la institución y de las necesidades sociales; y con equipo, libros, docentes, y organización escolar de calidad.

1.2. Compromiso con el personal: Fomentar su inclusión en las actividades con iniciativa, responsabilidad, satisfacción, promoción mejorada de sus funciones, y continua actualización vinculada a los cambios sociales que existen.

1.3. Compromiso social: Mediante la inserción de los egresados en actividades de los sectores públicos y privados, para dar respuesta a los problemas de criminalidad, riesgo, y condiciones criminógenas.

2. Importancia de la investigación para el desarrollo de la responsabilidad social en la criminología

El tema de la investigación es menester en múltiples áreas del conocimiento para la comprensión de los fenómenos del entorno (Quintana García, Benavides Velasco, y Marchante Lara, 2015) en el cual estamos inmersos seres humanos, otras formas de vida, la naturaleza, y los aspectos tecnológicos, entre otros; es decir, se busca percibir y descifrar el todo en las cosas, e ir más allá con tal información para poder incidir y transformar (Cantú Mendoza, 2015) como fin último para el mejoramiento de las condiciones que se tengan. "La investigación entonces se relaciona con todas las áreas epistemológicas que formulan la problemática de los delincuentes y el delito [...]” (Acosta Muñoz, 2017, p. 60). 
En el particular de la investigación enfatizada al campo de lo criminal, este suceder se aborda desde múltiples parámetros, teniendo parcelas de enfoques diversos con la psicología, el derecho, el trabajo social, la sociología, la estadística, la historia, la educación, entre otros, con prospectiva (Acosta Muñoz, 2017), cuyo objetivo es el de identificar, entender y conocer la dinámica de los complejos hechos humanos y sociales que conducen a los cambios y las influencias conjugadas que tienen en el contexto, para visualizar los efectos negativos y actuar con antelación.

Con aquellos conocimientos conjugados (Escobar Marulanda, 2006), se logran las necesarias interpretaciones de la conducta criminal en sus variadas expresiones, que, en general, sirven para generar un conocimiento que pueda ser utilizado por los planificadores de políticas públicas, gobernantes, o para el sector empresarial, y así, reducir el riesgo en el impacto social y económico que estas expresiones provocan; es decir, la comprensión de los fenómenos facilita o pretende establecer recaudos, o sea, se "considera al conocimiento como medio de bienestar posible del ser humano” (Massé, 2007, p. 297).

Tres elementos son los que a continuación se proponen como parte de la responsabilidad social de los centros de enseñanza de la criminología para basificar el desarrollo e investigación, siendo esta última el campo en el que convergen las tan variadas ópticas de interpretación de la criminalidad (Escobar Marulanda, 2006), abordadas en las áreas que han tomado por su objeto de estudio este problema, y cuyo incremento en su corpus teórico permiten auxiliar a la justicia como unidad de análisis (Acosta Muñoz, 2017):

2.1. Postular que la criminología mejore sus sistemas de investigación y de respuesta a los problemas sociales, económicos, laborales, familiares, juveniles, de adicciones, penitenciarios, entre tantos, que repercutan en más complicaciones (Acosta Muñoz, 2017). Con dichas investigaciones, proponer los mecanismos de intervención para hacerles frente tal y como lo establecen los objetivos de la misma cri- 
minología, y cuyo menester es plasmado por casi todas las ciencias humanas y sociales. "La investigación criminológica, en últimas, debe estar vinculada a las respuestas sobre la problemática que actualmente vive nuestra sociedad" (Acosta Muñoz, 2017, p. 65).

2.2. Incrementar el grosor teórico que existe, correspondiente a corrientes, autores, escuelas, pero sobre todo, catapultar a la criminología a generar teorías propias que nazcan de sí misma en un proceso transformador transdisciplinar, y con ello, hacer de ésta un cuerpo de conocimientos depurados, más allá de trabajar en la mera centralización de teorías circunferenciales al crimen, delito, violencia, antisocialidad, sociopatología, entre otros, que aportan saberes propios de su campo de estudio.

2.3. Es en el campo aplicativo donde se logra el proceso de aprender haciendo y aprender a hacer (Delors, 1996), para sistematizar los resultados y aportar a ese cuerpo de conocimientos en criminología, "investigar desde la práctica es el único camino para conocer en el terreno las particularidades" (Acosta Muñoz, 2017, p. 58) de los problemas que debemos abordar para procurar resolver. No existen laboratorios experimentales para la criminología, pero el más grande laboratorio se encuentra en la sociedad, donde de manera espontánea ${ }^{2}$, surgen factores que contribuyen a las expresiones criminales (de ese modo la experiencia consiste en el aprendizaje basado en problemas y en el aprendizaje basado en proyectos) (Barffusón, 2015), saber por qué los sujetos eligieron o se inclinaron por el mundo criminal, sus motivaciones o desmotivaciones; identificar y explicar son palabras clave para proponer mecanismos de prevención, control, reconstrucción de las condiciones deterioradas y de riesgo, de tal manera "que forje críticamente en el investigador una posición analítica de sus hallazgos investigativos" (Acosta Muñoz, 2017, p. 58).

Con lo anterior, el objetivo es mejorar el modelo de trabajo tradicional que se lleva a cabo en la criminología, el cual se ha centrado en recopilar teorías, más que desarrollarlas, lo que le ha autogene-

2 Sin querer pensar negativamente que los políticos y empresarios lanzan factores de riesgo que problematizan a los ciudadanos. 
rado debilidad ante ciencias con igualdad de tiempo de nacimiento (psicología, sociología y antropología), y más aún, ante ciencias que tienen siglos de desarrollo sistematizado (derecho y medicina). Los problemas sociales son cambiantes, y por ende, el conocimiento y las prácticas también deben evolucionar para prever y responder a ellos; conociendo el proceso que atraviesan hacía la criminalización, hay que "poner a prueba con la realidad las posibles soluciones a problemas específicos” (Acosta Muñoz, 2017, p. 58), en un marco de criminologías especializadas sobre temas que son demandantes de atención específica, particular y no general.

3. La especialización de la criminología: Otra responsabilidad latente para los centros de educación

El actual quehacer de los centros universitarios "se centra en orientar la formación general y especializada de cada estudiante” (Iñigo Bajos y Sosa Castillo, 2015: 168), aunque la realidad da ciertas conclusiones opuestas. En el panorama actual de la criminología, se acude a todo aquel conocimiento que, de manera metódica, proporcione enfoques que permitan identificar objetos y sujetos de estudio, para caracterizarlos, explicar su origen y desarrollo en un proceso de criminalización o que ya se desenvuelva socialmente en actos tipificados que dañan. Cada expresión de la criminalidad conlleva a un estudio interpretativo de ángulos que se aportan del derecho, la psicología, la antropología, entre otras. De esta manera, la criminología en su función concentradora, podría generar como resultado un embudo de explicaciones y un conocimiento integrado sobre los fenómenos criminales.

Cada campo del conocimiento que ha abordado una interpretación de la criminalidad ha evolucionado a un punto en el que se logra conocer el problema y aportar a su visión desde un enfoque biológico, psicológico, sociológico, económico, político, victimológico, jurídico, histórico, entre otros. Cada uno de estos campos mide el impacto que tiene lo criminal en diversos sectores de la vida social y particular. 
"Es necesario prever la evolución de la criminología y cómo otros saberes han dado su propia versión con enfoques analíticos para explicar la criminalidad” (Acosta Muñoz, 2017, p. 59).

Este aspecto, ligado a la tarea de investigación, buscaría provocar el desarrollo de la especialización de la criminología en problemas cuyas particularidades demandan interés de los investigadores, quienes a la par de su surgimiento, desarrollan explicaciones como producto de su estudio, y un nexo como propuesta de su solución, no tanto como una respuesta represiva que concluya en una pena privativa de libertad, sino en una función integradora y reconstructora de aquello dañado en la sociedad (Acosta Muñoz, 2017). De tal modo, se establecen vínculos entre:

\section{Figura 1. Vínculos investigación y desarrollo}

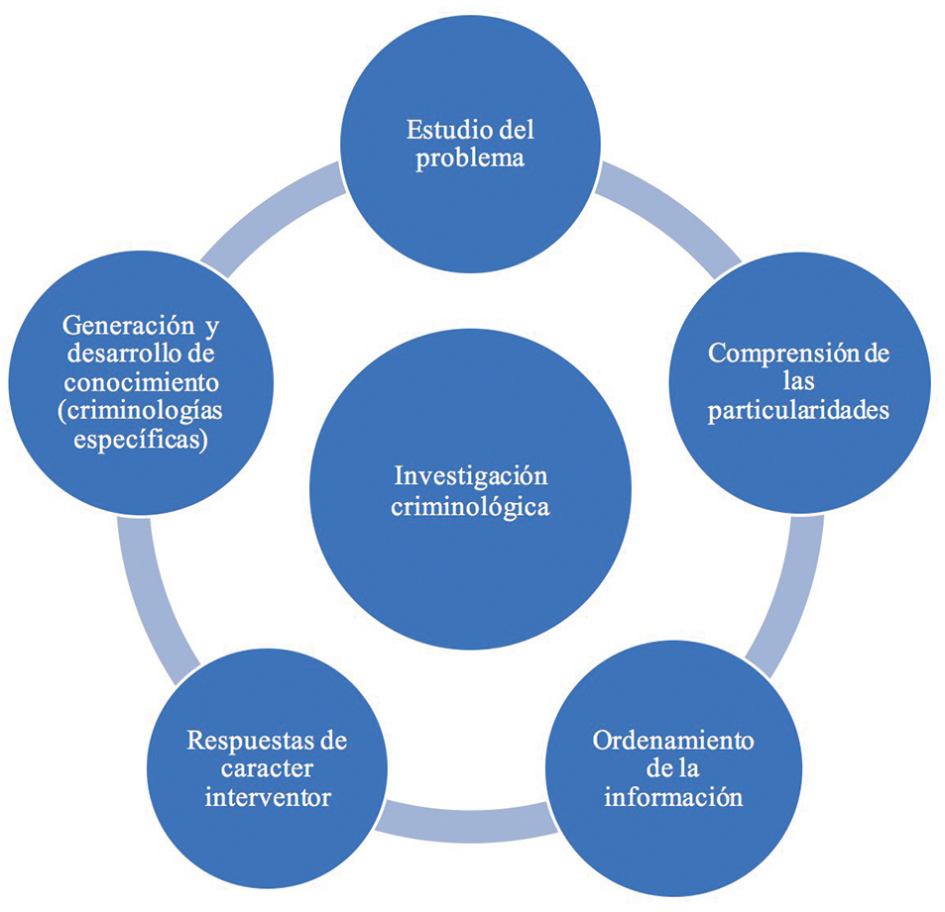

Fuente: Elaboración propia. 
Así las cosas, el desarrollo teórico de la criminología conduce a ramas "subdivididas en múltiples recortes para, pretendidamente, ir dando cuenta de los muchos campos [...]; casi siempre a partir de cuerpos teóricos ya construidos, y con ellos se va aportando conocimiento sobre algunos campos” (Massé, 2007, p. 305). En ese sentido, esta área de conocimiento propende por la producción de soluciones avanzadas que afronten las graves expresiones de la criminalidad, como paralelamente se da en el caso de la investigación, respecto del crecimiento del corpus intelectual, que permitirá mejorar los resultados, generando áreas de salidas profesionales que empoderan la pertinencia social (Cantú Mendoza, 2015). "Este tipo de investigación persigue desarrollar habilidades cognitivas, técnicas y sociales, para entender la práctica comprensiva del delincuente” (Acosta Muñoz, 2017, p. 60). En la medida de su pertinencia interna y externa, estará el valor de la criminología para lograr la incidencia y transformación social (Barffusón, 2015).

\section{Gráfica 2. Salidas de oportunidad en la investigación}

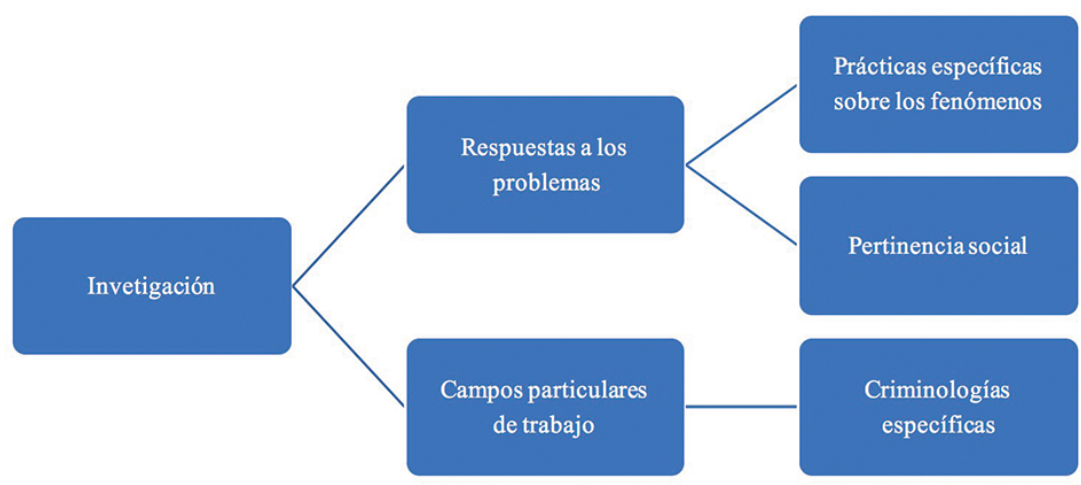

Fuente: elaboración propia 
El tema fundamental en la investigación para la especialización en asuntos de criminología es el que versa en relación con el marco teórico, los conceptos, los términos, el método, los objetos de estudio, los sujetos de estudio, los contenidos, las características, las particularidades, las definiciones, la evolución del problema, las respuestas de solución, los dilemas, los paradigmas, las formas de expresión, las salidas laborales, la pertinencia externa, y el impacto social.

La investigación asume y permite conocer el contenido en el que se desenvuelve la criminalidad, por lo que una especialización en la materia permitirá diferenciar entre la criminología tradicional de enfoque constelativo e interdisciplinar, de las criminologías especializadas con enfoque de reflexión epistemológica, de verificación y de validez de resultados, para crear puentes entre conocimientos que permitan alcanzar o llegar a un desarrollo innovador. "No obstante, existe muy poca teorización sobre los mismos si se asume la envergadura de la problemática” (Massé, 2007, p. 305).

\section{Conclusiones}

Los retos básicos para la apropiación de una criminología están a la vista, y son tres sus elementos, a saber: El primero, el valor social de la criminología está en la capacidad de resultados que den sus profesionales; el segundo, el desarrollo económico y profesional está ligado a las salidas profesionales que éste tenga, con base en su impacto social, especialización y capacidad de investigación y de respuesta a los problemas sociales de la criminalidad, y el tercero, que abarca los dos primeros, la responsabilidad social de los centros de educación en criminología, a partir de la cual se desprenden dos áreas, una, como instancias que forman personas con sentido de compromiso a los problemas de la sociedad, formando profesionales con capacidad de identificar, entender, proponer cambios, y la segunda, que es un compromiso propio de los centros de educación superior, y consiste en tanto producir y arrojar al mercado laboral personas ca- 
pacitadas en la criminología, como generar y propiciar el estudio de las sociedades, para dar respuesta a sus necesidades.

Los alcances para la criminología y el criminólogo, de comienzo, están revestidos por los centros educativos, pues éstos dan los conocimientos básicos para desarrollar el presente y el futuro hacia la comprensión y la transformación de la sociedad. El criminólogo es parte de un proceso de reconstrucción del tejido social, ya que este profesional puede aportar respuestas a los conflictos sociales desde las esferas que comprende su formación educativa, mediante el análisis de los fenómenos con reflexión epistemológica de las áreas de las que se nutre, con conocimientos propios de la criminalidad, vista desde enfoques múltiples, que en la capacidad aglutinadora de la criminología da una visión holística en interpretaciones y en propuestas e intervenciones. 


\section{Referencias bibliográficas}

Acosta Muñoz, D. (2017) "Investigación criminal en el medio penitenciario", en Investigación Criminal, vol. 1, n. ${ }^{\circ}$ 1: 58-69.

Barffusón, R. (2015) "La responsabilidad social de las universidades contemporáneas”, en: Cantú Mendoza, R. (coord.) La Responsabilidad Social de las Universidades Contemporáneas: 115-142. Universidad Autónoma de Nuevo León y Editorial Ítaca.

Cantú Mendoza, R. (2015) "Nuevos retos a la gestión de la educación superior en México”, en: Cantú Mendoza, R. (coord.) La Responsabilidad Social de las Universidades Contemporáneas: 13-38. Universidad Autónoma de Nuevo León y Editorial Ítaca.

Delors, J. (1996) La Educación encierra un tesoro. Grupo Santillana de Ediciones.

Escobar Marulanda, G. (2006) "La enseñanza en criminología. Algunas consideraciones”, en Revista Electrónica de Ciencia Penal y Criminología, vol. 8: 1-8. http://criminet.ugr.es/recpc/08/recpc08-r2.pdf

Gaete Quezada, R. (2015) “El gobierno y la gestión universitaria como ámbito de aplicación de la responsabilidad social”, en: Cantú Mendoza, R. (coord.) La Responsabilidad Social de las Universidades Contemporáneas: 143-156. Universidad Autónoma de Nuevo León y Editorial Ítaca.

Giménez-Salinas, E. (2009) Libro blanco sobre el título de grado en criminología. https:/criminologiacys.files.wordpress.com/2017/08/libro-blanco-sobre-el-tc3adtulo-de-grado-en-criminologc3ada.pdf

Iñigo Bajos, E. y Sosa Castillo, A.M. (2015) "Mitos y objetivos de la responsabilidad social universitaria”, en: Cantú Mendoza, R. (coord.) La responsabilidad social de las universidades contemporáneas: 157-174. Universidad Autónoma de Nuevo León y Editorial Ítaca.

Massé, C. (2007) "El sistema de la educación en Luhmann desde una perspectiva crítica”, en: Cinta Moebio, nro. 30: 296-308. http:/www.facso.uchile.cl/ publicaciones/moebio/30/masse.pdf

Quintana García, C., Benavides Velasco, C.A., y Marchante Lara, M. (2015) "Responsabilidad social en el sistema universitario español. Compromisos de las universidades”, en: Cantú Mendoza, R. (coord.) La Responsabilidad Social de las Universidades Contemporáneas: 175-192. Universidad Autónoma de Nuevo León y Editorial Ítaca.

Vences Esparza, A., Juárez Villalobos, G., y Flores Alanis, I.M. (2015) "La vinculación universitaria en el marco de la responsabilidad social en la UANL. Apuntes para redimensionar esta función”, en: Cantú Mendoza, R. (coord.) La Responsabilidad Social de las Universidades Contemporáneas: 67-86. Universidad Autónoma de Nuevo León y Editorial Ítaca. 